\title{
Environmental Equipment for Classic SEM Enabling Investigations of Dielectric and Wet Surfaces
}

\author{
W. SŁóWKO* AND M. KRYSZTOF \\ Faculty of Microsystem Electronics and Photonics, Wrocław University of Technology \\ Janiszewskiego 11/17, 50-372 Wrocław, Poland
}

\begin{abstract}
To extend capabilities of the classic scanning electron microscopes toward the variable pressure/environmental technique, the authors designed the vacuum and detection system in the form of a simple attachment which does not need any changes in an original structure of the instrument. The main part of the system is the vacuum and detection head, combining the intermediate vacuum chamber and electron detectors of chosen kinds. The low vacuum secondary electron detector extracts electrons through the lower throttling aperture and is designed in the form of an unipotential electrostatic lens with the anode covered with scintillator. It can work from pressures exceeding $10 \mathrm{hPa}$ to high vacuum, i.e. in the whole range of the scanning electron microscopy applications for almost all possible samples. The low vacuum secondary electron detector may be also made in a miniaturised autonomous form equipped with a photomultiplier or a semiconductor photodetector. These designs are destined for low vacuum scanning electron microscopy where the intermediate chamber is arranged inside an objective lens.
\end{abstract}

PACS: 41.85.-p, 52.80.Dy

\section{Introduction}

Classic scanning electron microscopes (SEM) are vacuum instruments which disqualify insulator specimens or those with high vapour constituents like water. These limitations can be overcome in variable pressure and environmental SEM (VP/E SEM) operating at elevated pressure in the sample chamber, while the electron optical column is maintained at high vacuum [1]. This implies differential pumping of the two regions which have to be separated by an intermediate chamber with vacuum of the order of $10^{-2} \mathrm{hPa}$, limited by two pinhole throttling apertures preventing intensive gas flow from the sample chamber to the high vacuum side. A problem inherent in the VP/E SEM technique is the detection of the secondary electron (SE) signal. As a rule, this is done in the sample chamber, and consists in collecting SEs multiplied by ionising collisions with gas molecules [1] or alternatively detecting photons produced by atoms excited in the collisions [2].

To extend capabilities of the classic instrument toward the VP/E technique, the authors supplemented it with the two mentioned units [3], designed in the form of a simple attachment, which can be mounted to a classic SEM, without changes in its original structure. The equipment contains an original electron detection system which can work both in high vacuum and at the elevated gas pressure exceeding $10 \mathrm{hPa}$.

\section{Structure of the equipment}

The system (Fig. 1) can be mounted in a classic SEM, without changes in its original structure. The main part

* corresponding author; e-mail: witold.slowko@pwr.wroc.pl of the system is the vacuum and detection head, combining the intermediate chamber and electron detectors of chosen kinds. For instance, the vacuum and detection head shown in Fig. 2 contains two types of electron detectors. At the end of the main light pipe (6) on the head axis, a low vacuum SE detector (1) is arranged. It can work both at pressures exceeding $10 \mathrm{hPa}$ and in high vacuum, so it could be the sole SE detector for the whole range of the SEM applications. Additionally, this detector may also be combined with a backscattered electron detector. However, a body of the intermediate chamber (2) restricts the stage tilt angle and a small opening of the throttling aperture limits the maximum view field, so the head has been made movable to be shifted aside with one move of a lever when vacuum is good. Simultaneously, a pusher (8) opens an inlet valve (9) to an auxiliary SE detector of the Everhard-Thornley type, connected to the same photomultiplier (7) as the low vacuum one. Besides, the head is equipped with a ball-socket joint enabling its angular movements up or down and to both sides.

\section{Axially symmetric SE detector}

The authors arranged a scintillator detector inside the intermediate chamber where the pressure level is low enough to apply voltages of a few $\mathrm{kV} \mathrm{[4].} \mathrm{The} \mathrm{detector} \mathrm{ex-}$ tracts SEs through the lower throttling aperture. Three electrodes of the detector system (Fig. 3a), i.e. a throttling aperture (1), an anode (2) covered with scintillator (2a) and a screening tube (3) create a miniature electrostatic unipotential electron lens, placed inside a light pipe $(4,4 \mathrm{a}, 4 \mathrm{~b})$ leading to a photomultiplier. The anode bias causes strong electric field penetrating through the throttling aperture to extract SEs generated from the sample. 


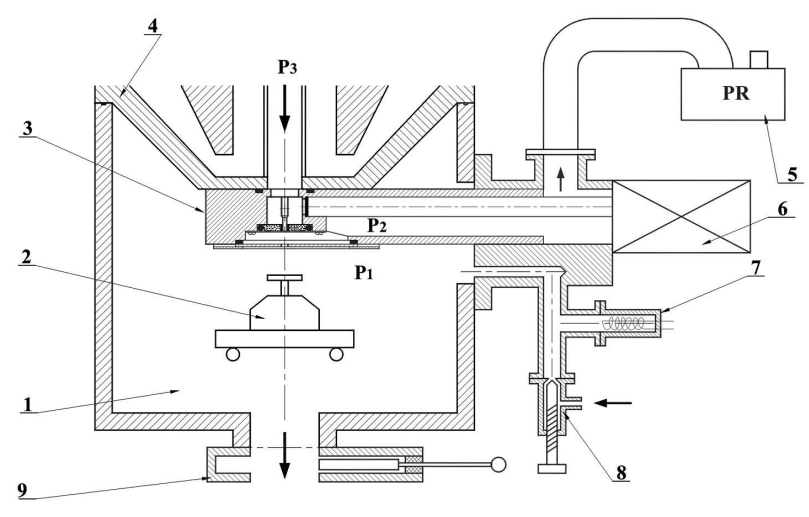

Fig. 1. Scheme of the intermediate vacuum and detection system (1 - sample chamber, 2 - sample holder, 3 - intermediate vacuum and detection head, 4 - objective lens, 5 - rotary pump, 6 - photomultiplier block, 7 - vacuum meter gauge, 8 - dosing valve, 9 - gate valve, $P_{1}, P_{2}, P_{3}$ - pressures in the sample chamber, the intermediate chamber and the electron optical column respectively, PR - rotary pump).

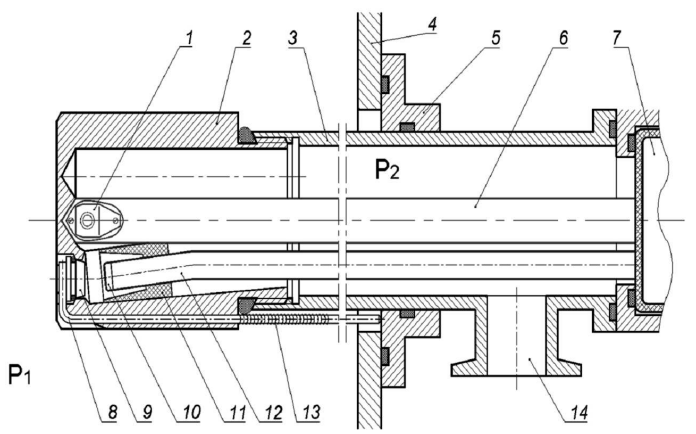

Fig. 2. Schematic section of the vacuum and detection head: 1 - low vacuum SE detector, 2 - intermediate chamber, 3 - connecting pipe, 4 - sample chamber wall, 5 - sealing block, 6 - main light pipe, 7 - photomultiplier, 8 - pusher, 9 - inlet valve, 10 scintillator, 11 - fixing insulator, 12 - auxiliary light pipe, 13 - spring, 14 - nipple.

When the pressure in the sample chamber is high enough, the anode field supplemented with that generated by the throttling aperture bias causes an ionising avalanche multiplying the electron signal emitted from the sample [5]. SEs focused on the throttling aperture flow into the intermediate chamber where they are captured by the anode electric field to gain a high kinetic energy converted into light signal on the scintillator layer. Light is transported to the photomultiplier to be converted back into electric signal and amplified.

Usually, a pressure in the intermediate chamber is low (about $0.1 \mathrm{hPa}$ or less), so in this region the gas amplification is small and the detector works similarly to the classic $E-T$ scintillator detector. There is also a possibility to work at higher pressures in the intermediate chamber, approaching $1 \mathrm{hPa}$. In this case, the electron

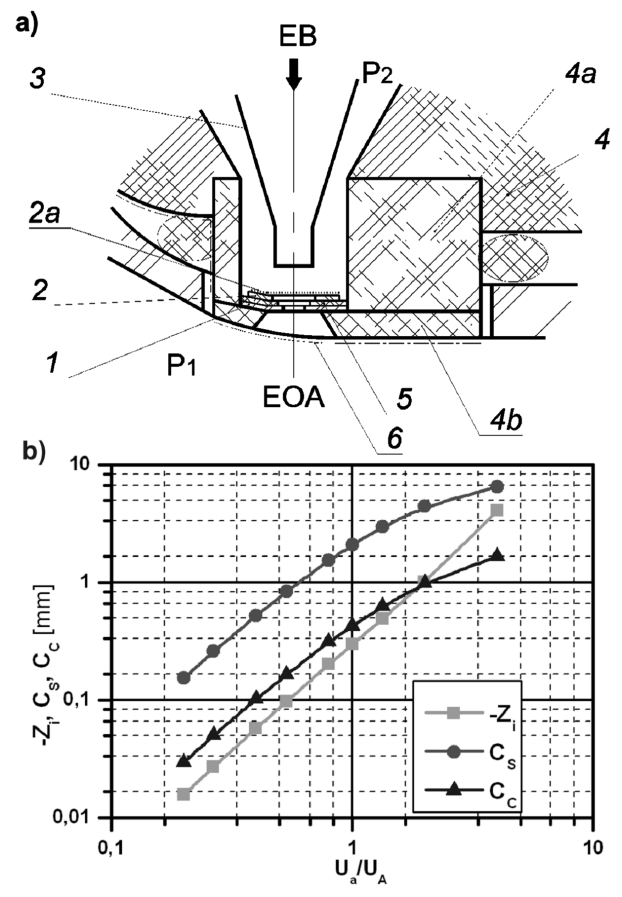

Fig. 3. Detector system: (a) scheme (1 - throttling aperture, 2 - anode, $2 \mathrm{a}$ - scintillator, 3 - screening tube, 4, 4a, 4b - light pipe elements, 5 - insulating separator, 6 - screening layer, $E B, E O A$ - electron beam, electron optical axis, $P_{1}, P_{2}$ - pressures in the sample and intermediate chamber), (b) coefficients of spherical $C_{\mathrm{s}}$ and chromatic $C_{\mathrm{c}}$ aberrations and the objective lens focus position $Z_{i}$, vs. the anode voltage $U_{\mathrm{a}}$ to the accelerating voltage $U_{\mathrm{A}}$ ratio.

signal can be multiplied in ionising processes and relaxations of excited particles may be a source of the light signal.

\section{Electron optical properties of the detector}

It was mentioned above that electrodes of the SE detector create a miniature electrostatic unipotential electron lens which affects the primary electron beam additionally to the electron optical column. Out of many possible configurations of the detector lens the one with the disc anode was shown schematically in Fig. 3a. It is obvious that the detector influence should not spoil the beam quality and a final resolution of the SEM. First of all, the lens structure should preserve strictly a rotational symmetry to avoid astigmatism and other asymmetry errors. However, the SEM resolution is mainly limited by spherical and chromatic aberrations represented by the coefficients $C_{\mathrm{s}}$ and $C_{\mathrm{c}}$, respectively. Besides, the detector lens adds its focusing power to the electro-optical column job, which should be compensated by a proper shift of the objective lens focus $Z_{i}$ in order to obtain the beam concentrated on the sample stage.

The electron optical properties of the detector lenses were investigated numerically with the use of the two 


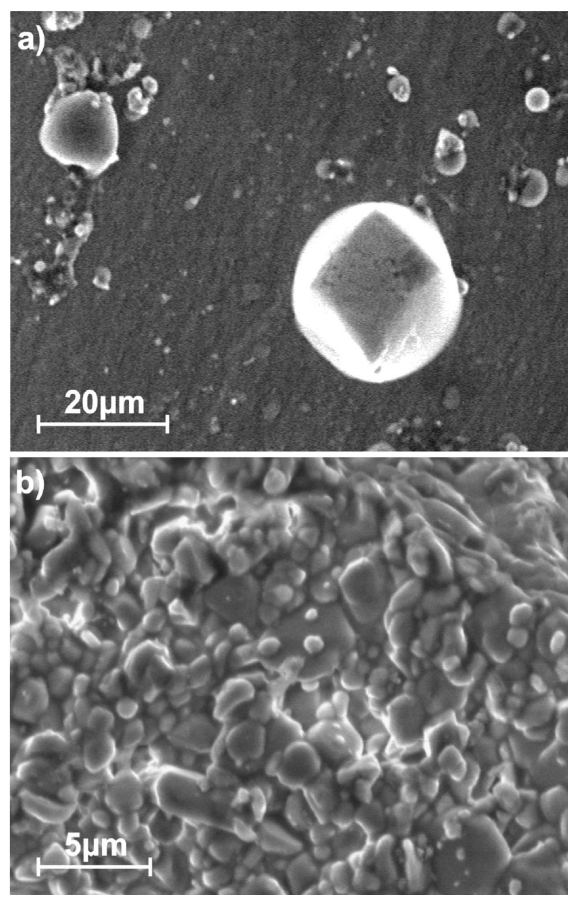

Fig. 4. Experiments with liquid water and dielectric samples (MR11 - own design SEM, $U_{\mathrm{a}}=15 \mathrm{kV}$ ): (a) $\mathrm{NaCl}$ crystal and dust particles deposited on an Al foil covered with droplets of water $\left(\approx 1{ }^{\circ} \mathrm{C}\right.$, water, $\left.P_{1}=7 \mathrm{hPa}\right)$, (b) ceramics $\left(20^{\circ} \mathrm{C}\right.$, air, $\left.2 \mathrm{hPa}\right)$.

programs: electrostatic lens design (ELD) and electron optical properties (EPROP) [6]. The results of calculations made for the configuration shown in Fig. 3a are displayed in Fig. 3b in a form of diagrams of the spherical and chromatic aberration coefficients $C_{\mathrm{s}}, C_{\mathrm{c}}$ and the focus shift $Z_{i}$ as a function of the detector anode voltage $U_{\mathrm{a}}$ related to the e-beam acceleration voltage $U_{\mathrm{A}}$. The values of $C_{\mathrm{s}}$ and $C_{\mathrm{c}}$ concern axial asymptotic aberration coefficients related to the object plane $[6,7]$ which can be used for evaluation of dispersion rings with simplified formulae

$$
\begin{aligned}
& \Delta_{\mathrm{s}} \approx C_{\mathrm{s}} \alpha_{0}^{3}, \\
& \Delta_{\mathrm{c}} \approx C_{\mathrm{c}} \alpha_{0} \Delta U_{\mathrm{A}} / U_{\mathrm{A}},
\end{aligned}
$$

where $\alpha_{0}$ is the aperture angle at the object side.

The diagrams show that aberration coefficients of the miniature detector lens depend strongly on the relation of accelerating voltages $U_{\mathrm{a}} / U_{\mathrm{A}}$ but preserve lower values than the typical objective lens does in the whole range of calculations, i.e. $C_{\mathrm{s}}<5 \mathrm{~mm}, C_{\mathrm{c}}<2 \mathrm{~mm}$ for $U_{\mathrm{a}} / U_{\mathrm{A}}<4 \mathrm{~V} / \mathrm{V}$. Also the focus shift $Z_{i}$ of the objective lens is very small and can be easily compensated by the lens excitation.

\section{Experimental}

Numerous experiments conducted by the authors proved that a classic SEM equipped with the described attachment is capable of working not only under high vacuum conditions but also fulfils the functions of $\mathrm{VP} / \mathrm{E}$ SEM imaging perfectly almost all possible samples from insulators to liquid water (Fig. 4). Ions generated in electron collisions with gas molecules recombine with electrons deposited by the scanning beam on non-conducting surfaces which enables investigations of dielectric samples without conductive coatings. On the other hand, samples containing liquid water like suspensions and slurries or biological samples can be observed on the Peltier stage cooled to $\approx 0{ }^{\circ} \mathrm{C}$. This reduces the water vapour pressure necessary to keep the sample in a natural state to $\approx 5 \mathrm{hPa}$, and facilitates imaging of light samples at low accelerating voltages.

\section{Conclusions}

General principles and properties of the detector are like the standard Everhart-Thornley detector. Basically, it does not need gas ionisation, which makes the detector system fast and universal with regard to the vacuum range (Fig. 2), i.e. from high vacuum to the pressures exceeding $10 \mathrm{hPa}$. The detector head height limiting the working distance $W D$ may be smaller than $10 \mathrm{~mm}$, while the distance to the sample traversed by the primary electron beam at the elevated gas pressure may be shorter than $1 \mathrm{~mm}$. This allows a low accelerating voltage without scattering the electron beam, which improves the contrast of light element samples, especially biological ones. The low vacuum SE detector may be also made in a miniaturised autonomous form equipped with a photomultiplier or a semiconductor photodetector. These designs are destined for low vacuum SEMs where the intermediate chamber is arranged inside an objective lens.

\section{References}

[1] G.D. Danilatos, Adv. Electron. Electron Phys. 71, 109 (1988).

[2] G.D. Danilatos, Adv. Electron. Electron Phys. 78, 1 (1990).

[3] W. Slówko, M. Krysztof, in: Proc. Microscopy Conf. MC2009, Vol. 1, Eds. G. Kothleitner, M. Leisch, Verlag der Technischen Universitat Graz, Graz 2009, p. 227.

[4] Patent application published by PCT, no. WO $2010 /$ 008307, A2, 2010.

[5] B.L. Thiel, I.C. Bache, A.L. Fletcher, P. Meredith, A.M. Donald, J. Microsc. 187, 143 (1997).

[6] Description of ELD and EPROP programs: http://www. lencova.cz .

[7] B. Lencová, M. Lenc, Optik 97, 121 (1994). 